Background Brazil invests more in scientific research than any other Latin American country. Its Ministry of Health is a source of funding for research, along with other development agencies, and its Department of STDs, HIV/AIDS and Viral Hepatitis is one of the agencies that most strongly supports and promotes research in the field of STDs/HIV/AIDS in Brazil. However, it remained to be seen whether or not effective use is being made of scientific evidence during public health policy formulation and if it is being used to validate the government's decision-making process. The purpose of this study was to assess the use of scientific evidence produced by projects funded by the Department.

Methods An exploratory study was carried out that surveyed former directors of the Department. Questions were asked concerning the Department's institutional mission, support for research and technological development, use of the results of research projects, and use of these projects' scientific evidence during public health policy formulation.

Results Analysis of the data showed that the Department has promoted and encouraged research studies and technological development, in partnership with centres for excellence in research, institutes of higher education, and national and international development agencies, and has funded 220 projects since 2004 , resulting in 1,198 scientific publications. Despite these contributions to advances in scientific knowledge, the present study showed that incorporation of scientific evidence was limited to research the Department had specifically requested, such as economic and strategic analyses and studies on operational programmes.

Conclusion Strategies for integrating science-based evidence with its practical application should be identified. Science-based evidence should be disseminated, promoted and incorporated into the process of developing public health policies, strategies, and practises.

\section{P6.074 TAILORING CLINICAL KNOWLEDGE PRODUCTS FOR GUIDANCE ON ADDRESSING SEXUALLY TRANSMITTED INFECTIONS AND BLOOD-BORNE INFECTION (STBBIS) TO MEET THE DIFFERENT NEEDS OF FAMILY MEDICINE PRACTITIONERS IN CANADA}

doi:10.1136/sextrans-2013-051184.1227

'J Seto, ${ }^{10}$ Kapral, ${ }^{1} \mathrm{~T}$ Wong, ${ }^{1} \mathrm{M}$ Gale-Rowe, ${ }^{1} \mathrm{~A}$ Demers, ${ }^{1} \mathrm{~J}$ Dodds, ${ }^{2} \mathrm{~W}$ Fisher, ${ }^{3} \mathrm{R}$ Read, ${ }^{4} \mathrm{M}$ Steben. 'Public Health Agency of Canada, Ottawa, ON, Canada; ${ }^{2}$ University of Western Ontario, London, ON, Canada; ${ }^{3}$ University of Calgary, Calgary, AB, Canada; 4/nstitut national de santé publique du Québec, Montréal, OC, Canada

Background Moving clinical guidelines into practise requires the development of clinical knowledge products to aid family practitioners. A variety of product types and formats may need to be used to suit different needs by career-stage.

Methods To evaluate the sexually transmitted and blood-borne infections (STBBI) knowledge product needs of practitioners, information was elicited from a convenience sample $(\mathrm{N}=207)$ of family medicine practitioners at a national Canadian family medicine forum in Toronto between November 15 and 17, 2013. Analysis was stratified by years of practise to identify ways to reach different generations of practitioners concerning STI and HIV clinical recommendations. The Fishers exact and chi-square test was used to determine statistical significance. Data was collected on preferences on clinical aids; methods used to update clinical knowledge; and preferred formats of accessing STI guidelines.

Results All practitioners expressed preferences for concise clinical aids such as algorithms (64.2\%) and abbreviated pocket guides $(35.3 \%)$, in addition to prompts on electronic medical records $(32.9 \%)$. On-line training and education were preferred by earlycareer practitioners ( $<5$-yrs: $53.3 \%)$, compared to their mid-to-late career $(\geq 5 y r s)$ counterparts $(26.1 \% ; p=0.004)$. More mid-to-late- career practitioners preferred classroom style workshops $(73.9 \%)$ than their early-career counterparts $(46.7 \% ; p=0.004)$. There were differences $(p=0.04)$ by years of practise concerning guideline format preferences. Early-career practitioners preferred mobile applications (38.5\%) compared to hard-copy materials (17.6\%). Mid-to-late-career practitioners appeared to prefer hard-copy materials $(31.2 \%)$ in addition to mobile applications (37.7\%).

Conclusions These findings demonstrate generational differences in preferred formats and methods of accessing STI and HIV clinical information; public health programmes may wish to deliver information using different approaches to reach different practitioners.

\section{P6.075 ATTEND TO THE "SMALL P" POLICY ISSUES: STATE POLICY ISSUES PREVENTING EFFECTIVE CERVICAL CANCER EFFORTS}

doi:10.1136/sextrans-2013-051184.1228

'B E Meyerson, 'C A Lawrence, ${ }^{2} \mathrm{~J}$ S Smith. 'Indiana University School of Public Health-Bloomington, Bloomington, IN, United States; ${ }^{2}$ UNC Gillings School of Global Public Health, Chapel Hill, NC, United States

Background We know little about policy issues at state level that impede cervical cancer efforts in the US. This study identifies and explores state policy issues in cervical cancer to inform future policy and programme strategies.

Methods Sixty minute key informant interviews were conducted with 15 professionals working in and around cervical cancer: federal and state government breast and cervical cancer programmes; women's health and cancer control; national advocacy organisations engaged in sexual health and cancer; legislative affairs; researchers working in cervical cancer intervention and evaluation; state coalitions engaged in cervical cancer efforts; and partners from industry working on diagnostics, therapeutics or vaccines.

Results State policy issues included health system complexity; lack of state level cervical cancer programme and policy coordination, and socio-organisational culture issues. System complexity was fueled by disparate and uncoordinated financing, controlled by different state programmes/agencies. A paucity of state level coordination exacerbated by a lack of cervical cancer inter-agency/programme influence prevented comprehensive, streamlined systems of care. Despite recent efforts to coordinate statewide cancer planning, cervical cancer issues and actors were not active in state priority setting. Socio-organisational cultural issues prevented normalisation of HPV vaccine for boys and girls, as well as programme and practitioner adoption of recent screening recommendations focused on identifying and monitoring persistent HPV.

Conclusions Critical state policy issues are not 'big p' issues or laws, but 'small p' issues of implementation and unintended systems outcomes of policy. Cross-agency, statewide planning could streamline systems for comprehensive and coordinated funding and policy. Critical need to address state leadership vacuum for cervical cancer efforts. Increasing the policy acumen and skill set of cervical cancer related programmes and coalitions could facilitate change navigation. Success at this 'small p' policy level will help to make big strides toward removing structural barriers to cervical cancer efforts in the U.S.

\section{P6.076 OUALITY OF LIFE AND ITS RELATIONSHIP WITH THE CLINICAL ASPECTS PEOPLE LIVING WITH HIV/AIDS IN BRAZIL}

doi:10.1136/sextrans-2013-051184.1229

${ }^{1}$ R K Reis, ${ }^{2} \mathrm{E}$ Gir, ${ }^{3} \mathrm{M}$ T G Galvão. 'Nursing School of Ribeirão Preto, University of São Paulo, Ribeirão Preto-São Paulo, Brazil; ${ }^{2}$ Nursing School of Ribeirão Preto, University of São Paulo, Ribeirão Preto, Brazil; ${ }^{3}$ Professor of Nursing Course at Federal University of Ceará, Fortaleza, Brazil 
The objectives of this study were to evaluate the quality of life of people living with HIV/AIDS and its relationship with clinical factors. This cross-sectional analytical study was performed with a non-probabilistic sample, consisting of people living with HIV/ AIDS and undergoing clinical and outpatient treatment in two health services specialised in AIDS care in the municipal healthcare network of Ribeirão Preto, São Paulo, Brazil. Data collection was performed by means of individual interviews using a script for the sociodemographic and clinical characterization, and the HIV/AIDStargeted quality of life instrument (HATQoL). The participants were 228 individuals living with HIV/AIDS, 122 (53.5\%) men and $106(46.5 \%)$ women, with a mean age of 39 years. Regarding the clinical variables, it was identified that the participant's time of awareness regarding the HIV diagnosis was related to a poorer quality of life (OL) in several domains. The immunological condition is one of the variables that was related with a better $\mathrm{OL}$, as individuals with a CD4 cell count greater than 500 cells $/ \mathrm{mm}^{3}$ showed better means with statistically significant differences in five domains: overall activity, health worries, HIV mastery, life satisfaction, and medication worries. Furthermore, it was identified that individuals with AIDS had lower scores with significant differences in the domains: overall function and disclosure worries. Understanding the variables related to the OL of people living with HIV/AIDS is key, considering the chronic evolution of the infection, the possibility of treatment, greater survival and dealing better with the disease. The clinical variables should be continually monitored by the health team considering that the clinical and/or immunological condition is related to several aspects such as adherence to treatment, evolution of the infection, physical and mental health condition, as well as quality of life. 